Draft Version November 21, 2018

Preprint typeset using $\mathrm{LT}_{\mathrm{E}} \mathrm{X}$ style emulateapj v. 03/07/07

\title{
MASER EMISSION FROM SIO ISOTOPOLOGUES TRACES THE INNERMOST 100 AU AROUND RADIO SOURCE I IN ORION BN/KL
}

\author{
C. Goddi, L. J. Greenhill \\ Harvard-Smithsonian Center for Astrophysics, 60 Garden Street, Cambridge, MA 02138 \\ AND \\ C. J. Chandler \\ National Radio Astronomy Observatory, P.O. Box O, Socorro, NM 87801 \\ AND \\ E. M. L. Humphreys, L. D. Matthews* \\ Harvard-Smithsonian Center for Astrophysics, 60 Garden Street, Cambridge, MA 02138
}

AND

M.D. GraY

Jodrell Bank Centre for Astrophysics, Alan Turing Building, School of Physics and Astronomy, University of Manchester, Manchester M13 9PL

Draft version November 21, 2018

\begin{abstract}
We have used the Very Large Array (VLA) at $7 \mathrm{~mm}$ wavelength to image five rotational transitions $(J=1-0)$ from three $\mathrm{SiO}$ isotopologues towards Orion $\mathrm{BN} / \mathrm{KL}:{ }^{28} \mathrm{SiO} v=0,1,2$; and ${ }^{29} \mathrm{SiO}$ and ${ }^{30} \mathrm{SiO} v=0$. For the first time, we have mapped the ${ }^{29} \mathrm{SiO}$ and ${ }^{30} \mathrm{SiO} J=1-0$ emission, established the maser nature of the emission, and confirmed association with the deeply embedded high-mass young stellar object commonly denoted radio Source I. The ${ }^{28} \mathrm{SiO} v=0$ maser emission shows a bipolar structure that extends over $\sim 700 \mathrm{AU}$ along a northeast-southwest axis, and we propose that it traces a bipolar outflow driven by Source I. The high-brightness isotopic $\mathrm{SiO}$ maser emission imaged with a $\lesssim 0$ '! 2 resolution has a more compact distribution, generally similar to that of the ${ }^{28} \mathrm{SiO} v=1,2$ emission, and it probably traces bulk gas flows in a region of diameter $\lesssim 100$ AU centered on Source I. On small scales of $\lesssim 10 \mathrm{AU}$, however, compact ${ }^{29} \mathrm{SiO} /{ }^{30} \mathrm{SiO} v=0$ and ${ }^{28} \mathrm{SiO} v=1,2$ emission features may be offset from one another in position and line-of-sight velocity.

From a radiative transfer analysis based on a large velocity gradient (LVG) pumping model, we derive similar temperatures and densities for the optimum excitation of both ${ }^{29} \mathrm{SiO} /{ }^{30} \mathrm{SiO} v=0$ and ${ }^{28} \mathrm{SiO} v=1,2$ masers, significantly higher than required for ${ }^{28} \mathrm{SiO} v=0$ maser excitation. In order to account for the small-scale differences among the isotopologues $(v=0)$ and the main species $(v=1,2)$, follow-up radiative transfer modeling that incorporates non-local line overlap among transitions of all $\mathrm{SiO}$ isotopic species may be required.
\end{abstract}

Subject headings: line: profiles - masers - ISM: individual objects: Orion BN/KL - stars: formation

\section{INTRODUCTION}

The Orion Becklin-Neugebauer/Kleinman-Low $(\mathrm{BN} / \mathrm{KL})$ region $(D \sim 414 \pm 7 \mathrm{pc}$; Menten et al. 2007) is one of only three star forming regions (SFRs) known to exhibit maser emission from ${ }^{28} \mathrm{SiO}$ (Hasegawa et al. 1986; Zapata et al. 2008) and the only one to display emission from the major $\mathrm{SiO}$ isotopic species comprising ${ }^{16} \mathrm{O}:{ }^{28} \mathrm{SiO},{ }^{29} \mathrm{SiO},{ }^{30} \mathrm{SiO}$ (e.g., Goddi et al. 2009), and ${ }^{18} \mathrm{O}:{ }^{28} \mathrm{Si}^{18} \mathrm{O}$ (Cho et al. 2005).

Early single-dish and interferometric observations of $J=2-1(\lambda 3 \mathrm{~mm})$ and $J=1-0(\lambda 7 \mathrm{~mm})$ rotational transitions in the $v=1$ and $v=2$ vibrational states have shown similarities among spectral profiles and spatial distributions, possibly indicating that they arise in the same gas volumes. The vibrationally excited states have been known to be inverted since their first identification (Thaddeus et al. 1974; Buhl et al. 1974). Observations with the Very Large Array (VLA) first showed that the center of the $v=1(J=1-0) \mathrm{SiO}$ maser emis-

*Present address: MIT Haystack Observatory, Westford, MA 01886 sion coincides with the radio continuum source denoted Source I (Churchwell et al. 1987; Menten \& Reid 1995). Subarcsecond imaging at near- and mid-infrared wavelengths shows no infrared counterpart to Source I, which suggests it is a deeply embedded young stellar object or YSO (Gezari 1992; Greenhill et al. 2004b). High-angular resolution imaging with Very Long Baseline Interferometry (VLBI) in both $J=1-0$ and $J=2-1$ lines has shown that the $v=1$ and $v=2$ emission traces a protostellar wind and/or outflow expanding with a characteristic velocity $\lesssim 20 \mathrm{~km} \mathrm{~s}^{-1}$ at radii of $20-70 \mathrm{AU}$ from Source I (Greenhill et al. 1998; Doeleman et al. 1999; Greenhill et al. 2004a; Doeleman et al. 2004; Matthews et al. 2007; Matthews et al. in prep.).

Thermal ${ }^{28} \mathrm{SiO} v=0$ emission is a well-known tracer of shocks in protostellar jets (e.g., Nisini et al. 2007). After the discovery of $v=0 J=2-1$ (Buhl et al. 1975) and $J=1-0$ (Genzel et al. 1980) lines in Orion $\mathrm{BN} / \mathrm{KL}$, interferometric imaging demonstrated that the vibrational ground state emission is part maser and part thermal $(J=1-0$ : Chandler \& de Pree 1995; $J=2-1$ : Wright et al. 1995). The ${ }^{28} \mathrm{SiO} v=0$ traces a bipolar 
structure elongated northeast-southwest (NE-SW), that has been interpreted either as a rotating disk $(J=2-1$; Wright et al. 1995) or as a wide-angle bipolar outflow with characteristic speeds of $10-15 \mathrm{~km} \mathrm{~s}^{-1}(J=1-0$; Greenhill et al. 2004a).

The ${ }^{29} \mathrm{SiO}$ and ${ }^{30} \mathrm{SiO}$ isotopologues were first discovered towards Orion BN/KL in the $v=0 J=2-1$ line (Lovas et al. 1976; and Wolff 1980, respectively). Olofsson et al. (1981) first proposed maser action for the $\mathrm{SiO}(J=2-1)$ isotopic emission, based on observed (yearly) variability of individual Doppler components $\left({ }^{29} \mathrm{SiO}\right)$ or the asymmetric line shape $\left({ }^{30} \mathrm{SiO}\right)$. Baudry et al. (1998) confirmed maser action for the ${ }^{29} \mathrm{SiO} J=2-1$ transition via high-angular resolution $\left(1-3^{\prime \prime}\right)$ imaging, but there has been no conclusive evidence of maser action for the $J=1-0$ transition or for ${ }^{30} \mathrm{SiO}$ in either transition.

The physical origin of the isotopic emission is not yet well determined. Plateau de Bure Interferometer (PdBI) imaging shows that the centroids of ${ }^{29} \mathrm{SiO}$ emission $(J=2-1)$ in multiple velocity channels between -11 and $24 \mathrm{~km} \mathrm{~s}^{-1}$ arise $\lesssim 100 \mathrm{AU}$ from Source I, when observed with $1-3^{\prime \prime}$ resolution (Baudry et al. 1998). In contrast, the higher order $J=8-7$ transition of ${ }^{30} \mathrm{SiO}$, observed with the Smithsonian Submillimeter Array (SMA) and comparable angular resolution, is distributed over $\sim 1000$ AU, elongated NE$\mathrm{SW}$, as is the part-thermal, part-maser ${ }^{28} \mathrm{SiO} v=0$ emission (Beuther et al. 2005). Single-dish spectra for the ground-state ${ }^{29} \mathrm{SiO}$ and ${ }^{30} \mathrm{SiO} J=1-0$ transitions also exhibit line wings that are similar to those of ${ }^{28} \mathrm{SiO}\left( \pm 40 \mathrm{~km} \mathrm{~s}^{-1}\right)$, as recently reported by Goddi et al. (2009). These are broader than the velocity extent of vibrationally excited emission and invite speculation that they might trace moderately high-velocity components of the flows around Source I (Goddi et al. 2009).

Sub-arcsec resolution imaging is necessary to establish unambiguously the location and the nature (maser vs thermal) of the isotopic emission within the BN/KL region, in particular where the high-velocity isotopic emission arises within the $\mathrm{SiO}$ flow. With this purpose, we conducted simultaneous observations of the $v=0, v=1$, and $v=2 J=1-0$ transitions of ${ }^{28} \mathrm{SiO}$ and the $v=0 J=1-0$ line of the ${ }^{29} \mathrm{SiO}$ and ${ }^{30} \mathrm{SiO}$ isotopologues at $7 \mathrm{~mm}$ wavelength with the VLA. For the first time, we have mapped the ${ }^{29} \mathrm{SiO}$ and the ${ }^{30} \mathrm{SiO} \quad v=0 J=1-0$ lines, measured the proximity of the emission regions to Source I, and established the presence of population inversion. Simultaneous observation of $\mathrm{SiO}$ masers from three vibrationally excited transitions and three isotopologues, provide constraints on $\mathrm{SiO}$ excitation conditions in the surrounding medium.

The observational setup and data calibration procedures are described in $\S 2$. Spectral profiles and images of different maser transitions are presented in $\S 3$. In $\S 4$, we discuss the gas kinematics probed by each species (§4.1), the presence of maser action from ${ }^{29} \mathrm{SiO}$ and ${ }^{30} \mathrm{SiO}(\S 4.2)$, and the excitation conditions derived from preliminary radiative transfer modeling (\$4.3). Finally, conclusions are drawn in $\S 5$.

\section{OBSERVATIONS AND DATA REDUCTION}

\subsection{Observations}

TABLE 1

Parameters of Observations.

\begin{tabular}{cccccc}
\hline \hline Program & Date & $\begin{array}{c}\text { VLA } \\
\text { Conf. }\end{array}$ & $\begin{array}{c}\text { Transitions } \\
J=1-0\end{array}$ & $\begin{array}{c}\nu_{\text {rest }} \\
(\mathrm{MHz})\end{array}$ & $\begin{array}{c}T^{(2)} \\
(\mathrm{hr})\end{array}$ \\
\hline $\mathrm{AG776}$ & $2008 / 02 / 02$ & $\mathrm{~B}(13 \mathrm{~V})$ & ${ }^{28} \mathrm{SiO} v=0$ & 43423.858 & 2.3 \\
$\mathrm{AG776}$ & $2008 / 02 / 02$ & $\mathrm{~B}(13 \mathrm{~V})$ & ${ }^{28} \mathrm{SiO} v=1$ & 43122.080 & 4.6 \\
$\mathrm{AG776}$ & $2008 / 02 / 02$ & $\mathrm{~B}(13 \mathrm{E})$ & ${ }^{28} \mathrm{SiO} v=2$ & 42820.587 & 4.6 \\
$\mathrm{AG776}$ & $2008 / 02 / 02$ & $\mathrm{~B}(13 \mathrm{~V})$ & ${ }^{29} \mathrm{SiO} v=0$ & 42879.846 & 2.3 \\
$\mathrm{AG} 776$ & $2008 / 02 / 02$ & $\mathrm{~B}(13 \mathrm{E})$ & ${ }^{30} \mathrm{SiO} v=0$ & 42373.340 & 4.6 \\
$\mathrm{AG} 575$ & $2000 / 01 / 01$ & $\mathrm{~B}(19)$ & ${ }^{28} \mathrm{SiO} v=1$ & 43122.080 & 0.5 \\
$\mathrm{AG} 575$ & $2000 / 01 / 01$ & $\mathrm{~B}(19)$ & ${ }^{29} \mathrm{SiO} v=0$ & 42879.846 & 0.5 \\
$\mathrm{AG} 575$ & $1999 / 08 / 28$ & $\mathrm{~A}(10)$ & ${ }^{28} \mathrm{SiO} v=1$ & 43122.080 & 0.8 \\
$\mathrm{AG} 575$ & $1999 / 08 / 28$ & $\mathrm{~A}(10)$ & ${ }^{29} \mathrm{SiO} v=0$ & 42879.846 & 0.4 \\
\hline
\end{tabular}

Note. -

${ }^{1}$ Spacing of antennas: B-configuration has baselines of 0.1 to $11 \mathrm{~km}$. Aconfiguration has baselines of 0.5 to $35 \mathrm{~km}$. The number of antennas participating is in parentheses, trailed by "V" to indicate VLA and "E" to indicate EVLA. Prior to 2008, the number of participating antennas corresponds to the number equipped with high-frequency receivers. All setups placed either $v=1$ or $v=2{ }^{28} \mathrm{SiO}$ in one IF to enable high-accuracy calibration.

${ }^{2}$ Approximate on-source integration time.

Observations of the $\mathrm{SiO}$ emission from Source I were conducted at $7 \mathrm{~mm}$ using the Very Large Array (VLA) of the National Radio Astronomy Observatory (NRAO) ${ }^{1}$. We present data obtained on 2008 February 2, along with archival data from 1999 August 28 and 2000 January 1. The data from 2000 and 2008 were obtained while the VLA was in the B-configuration, yielding a resolution of $\sim 0.2$. The data from 1999 are from the A-configuration, giving a resolution of $\sim 0.5$. Table 1 summarizes the observational parameters of the different programs. The following description refers to the program observed in 2008.

We observed a total of five $J=1-0$ rotational transitions from three different $\mathrm{SiO}$ isotopologues: ${ }^{28} \mathrm{SiO}$ $v=0,1,2 ;{ }^{29} \mathrm{SiO} v=0$; and ${ }^{30} \mathrm{SiO} v=0$ (Table [1). To achieve high-accuracy astrometry, we cycled our observations among the various transitions, observing two at a time. We included emission from the $v=1$ or $v=2$ states of ${ }^{28} \mathrm{SiO}$ in each of the setups because these were strong enough to be used to calibrate the troposphere and system gain, thus facilitating detection of the weaker emission from the other isotopologues. At the time of the 2008 observations the VLA included 13 EVLA $^{2}$ antennas that were able to simultaneously observe two separate bandpasses (IFs) up to $2 \mathrm{GHz}$ apart. In contrast, the maximum separation between IFs allowable with the remaining (VLA) antennas was $\sim 400 \mathrm{MHz}$, depending on details of the available local oscillator settings. In order to optimize the use of the VLA and EVLA antennas, respectively, we therefore divided the array into two sub-arrays of 13 antennas each. The VLA antennas observed the ${ }^{28} \mathrm{SiO} v=1$ maser at $43122 \mathrm{MHz}$ in one IF (single polarization) and the ${ }^{28} \mathrm{SiO}$ and ${ }^{29} \mathrm{SiO} v=0$ lines at $43424 / 42880 \mathrm{MHz}$ alternately in the other IF (single polarization). The EVLA antennas observed the ${ }^{28} \mathrm{SiO} v=2$ maser at $42821 \mathrm{MHz}$ simultaneously with the

1 NRAO is a facility of the National Science Foundation operated under cooperative agreement by Associated Universities, Inc.

2 The Expanded Very Large Array is a joint USA, Mexico, and Canada project to greatly improve the observing capabilities of the VLA array by installing new electronics (fiber optic cables, large bandwidth receivers, new correlator, etc.) while keeping the existing infrastructure (antennas, railroad tracks, etc.). 
${ }^{30} \mathrm{SiO} v=0$ line at $42373 \mathrm{MHz}$ (all single polarization). For ${ }^{30} \mathrm{SiO} v=0$, a tuning offset of $2.83 \mathrm{MHz}$ was introduced alternately with the main tuning to enable a search for possible high-velocity emission (up to $70 \mathrm{~km} \mathrm{~s}^{-1}$ ), whose presence was suggested in earlier single-dish spectra (Goddi et al. 2009). No such emission was detected by the present observations. The total on-source integration times for the various $\mathrm{SiO}$ transitions are given in Table 1, and the frequency pairings used in the observing programs are summarized in Table 2. The ${ }^{28} \mathrm{SiO},{ }^{29} \mathrm{SiO}$, and ${ }^{30} \mathrm{SiO} v=0$ transitions were each observed with a $12.5 \mathrm{MHz}$ total bandwidth $\left(88 \mathrm{~km} \mathrm{~s}^{-1}\right)$ with a spectral resolution of $390.6 \mathrm{kHz}\left(2.7 \mathrm{~km} \mathrm{~s}^{-1}\right)$. The ${ }^{28} \mathrm{SiO} v=1,2$ emission was observed with a $6.25 \mathrm{MHz}$ bandwidth and $97.656 \mathrm{kHz}\left(0.68 \mathrm{~km} \mathrm{~s}^{-1}\right)$ channels (Table 2).

We added several continuum-mode setups to the 2008 observations to estimate any frequency dependent (i.e., delay) terms to the astrometry error budget that might affect the relative astrometry between the pairings of transitions observed in spectral-line mode. In particular, we performed observations of the respective ${ }^{28} \mathrm{SiO}$ maser $(v=1$ for the VLA subarray, $v=2$ for the EVLA subarray) in one IF (single channel, $6.25 \mathrm{MHz}$ bandwidth, dual polarization) together with observations in a $50 \mathrm{MHz}$ continuum channel in the other IF (also dual polarization), offset in frequency by $\pm 350 \mathrm{MHz}$ from the maser. The goal was to detect and image the $\mathrm{BN}$ object and use its position to align the various $\mathrm{SiO}$ emissions. Unfortunately these observations on the EVLA subarray failed, so we have aligned the $v=1$ and $v=2$ emissions with respect to each other using other techniques (Section 2.3)

We derived absolute flux calibration from observations of $3 \mathrm{C} 286\left(F_{\nu}=1.5 \mathrm{Jy}\right)$, and bandpass calibration from observations of $3 \mathrm{C} 84$. The phase offsets between the paired IFs were tracked through observations of QSO J0541-0541 every 25 minutes. The strong ${ }^{28} \mathrm{SiO} v=1$ and $v=2$ masers $(\gg 100 \mathrm{Jy}$ ) were used to track tropospheric phase fluctuations on 10-s timescales, as described in Section 2.2. All spectral-line observations were observed in a fixed-frequency mode, and Doppler corrections were applied during the data reduction.

Observations of ${ }^{29} \mathrm{SiO} v=0$ and ${ }^{28} \mathrm{SiO} v=1$ in 1999 and 2000 (Table 1) were made with setups similar to those used in 2008 for the VLA antennas. We adopted a 2-IF, single polarization mode, with bandwidths of $6.25 \mathrm{MHz}$. Monitoring of phase offsets between the IFs was accomplished with periodic observations of QSO J0541-0541, and bandpass calibration relied upon observations of QSO J0530+135.

\subsection{Calibration procedures}

Calibration and data reduction were carried out using the Astronomical Image Processing System (AIPS). First, the phase offset between the two simultaneouslyobserved IFs due to the electronics was removed, so that subsequent tropospheric calibration from the ${ }^{28} \mathrm{SiO} v=1$ and $v=2$ transitions could be applied directly to the IF containing the weaker line. The phase offset was determined from observations of J0541-0541 in the following manner. Using the $12.5 \mathrm{MHz}$ bandwidth IF, calibration solutions were determined for the short-term tropospheric phase fluctuations on J0541-0541 and applied to J0541-0541 in the $6.25 \mathrm{MHz}$ IF. A subsequent phase cal- ibration on the $6.25 \mathrm{MHz}$ IF using J0541-0541 and averaging over scan lengths $(\sim 2 \mathrm{~min})$ then gave the phase offset of this IF relative to the $12.5 \mathrm{MHz}$, and was applied to all sources in the dataset. Amplitude calibration was subsequently derived from J0541-0541, after correcting for the short-term tropospheric phase fluctuations. We also derived separately phase solutions from 2-minute scan averages of J0541-0541 and applied them to Source I. Corrections for the shape of the antennabased bandpasses were also applied, along with Doppler corrections.

Finally, corrections for fluctuations in the tropospheric and instrumental phase and amplitude were derived every 10 seconds from channel averages of the strong ${ }^{28} \mathrm{SiO}$ masers $\left(V_{\mathrm{LSR}}=-7.2\right.$ to $-3.1 \mathrm{~km} \mathrm{~s}^{-1}$ for the $v=1$ line, and -9.4 to $-5.3 \mathrm{~km} \mathrm{~s}^{-1}$ for the $v=2$ line) using selfcalibration and applied to the IF containing the weaker line. As a consequence, maps of the ground-state transitions are (independently) referenced to the positions of the ${ }^{28} \mathrm{SiO} v=1$ or $v=2$ emission.

Images were made of Source I using the AIPS task IMAGR with a "robust" parameter of 0 , resulting in a synthesized beam size of $\sim 0$ !' 2 for the B configuration data and $\sim 00^{\prime \prime} 05$ for the A configuration data. The images were 1024 pixels on a side with cell sizes 0 ! $^{\prime \prime} 02$ (2008) or $0{ }^{\prime \prime} 005$ (1999) and covered fields-of-view of $20^{\prime \prime}$ and $5^{\prime \prime}$, respectively. For the ${ }^{28} \mathrm{SiO}$ images the sensitivity is dynamic range limited by the sidelobes of the strongest emission peaks and thus varies among different frequency channels and different transitions. Table 3 summarizes the imaging results.

\subsection{Positional registration among different transitions}

Phase and amplitude self-calibration enable coherent integration and imaging but residual astrometric errors remain, due to the $\sim 1^{\circ}$ separation between calibrator (J0541-0541) and target. To remove frequency and sky position dependent errors (i.e., the residual delay error due to the atmosphere), we used as a position reference for registration the peak of the continuum emission from $\mathrm{BN}$, which is the strongest in Orion $\mathrm{BN} / \mathrm{KL}$ $\left(F_{\nu} \sim 25 \mathrm{mJy}\right.$ at $\left.7 \mathrm{~mm}\right)$ and only $\sim 10^{\prime \prime}$ from Source I. For that purpose, we calibrated the continuum-mode dataset from 2008 following a procedure similar to that described in Sect. 2.2 for the line-mode dataset. The band-averaged signal-to-noise ratio (SNR) for the $v=1$ and $v=2$ maser emission over $6.25 \mathrm{MHz}$ was high enough that the narrow band data enabled coherent integration and detection of $\mathrm{BN}$ in the broad band.

By directly comparing the positions of $\mathrm{BN}$ in bands tuned to the $v=1$ emission and offset by $\pm 350 \mathrm{MHz}$, we established the angular offset per $\mathrm{MHz}$ introduced by calibrating one band by another: $\Delta \alpha=0.007 \pm$ $0.003 \mathrm{mas} / \mathrm{MHz}$ and $\Delta \delta=-0.016 \pm 0.004 \mathrm{mas} / \mathrm{MHz}$. Unfortunately, the EVLA subarray tuned to the $v=2$ emission did not provide usable data in the continuum mode setups. We used the offset derived with the VLA subarray to correct the positions of all pairs of transitions with 1 mas accuracy.

Since the $v=1$ and $v=2$ transitions served as calibration references for different subarrays and were self-calibrated independently, without an EVLA detection of $\mathrm{BN}$ it is necessary to register them separately to each other. For that purpose we measured the intrinsic 
TABLE 2

FREQUENCY PAIRS IN THE OBSERVING SETUP

\begin{tabular}{llcccccc}
\hline \hline Prog. & Mode & IFA(-C) & $\begin{array}{c}\mathrm{BW} \\
(\mathrm{MHz})\end{array}$ & $\begin{array}{c}\mathrm{CW} \\
(\mathrm{kHz})\end{array}$ & $\mathrm{IFB}(-\mathrm{D})$ & $\begin{array}{c}\mathrm{BW} \\
(\mathrm{MHz})\end{array}$ & $\begin{array}{c}\mathrm{CW} \\
(\mathrm{kHz})\end{array}$ \\
\hline AG776 & Line & ${ }^{28} \mathrm{SiO} v=0$ & 12.5 & 390.6 & ${ }^{28} \mathrm{SiO} v=1$ & 6.25 & 97.7 \\
$\mathrm{AG776}$ & Line & ${ }^{29} \mathrm{SiO} v=0$ & 12.5 & 390.6 & ${ }^{28} \mathrm{SiO} v=1$ & 6.25 & 97.7 \\
$\mathrm{AG776}$ & Line & ${ }^{30} \mathrm{SiO} v=0$ & 12.5 & 390.6 & ${ }^{28} \mathrm{SiO} v=2$ & 6.25 & 97.7 \\
$\mathrm{AG776}$ & Cont. & $v=1+350$ & 50.0 & - & ${ }^{28} \mathrm{SiO} v=1$ & 6.25 & - \\
$\mathrm{AG776}$ & Cont. & $v=1-350$ & 50.0 & - & ${ }^{28} \mathrm{SiO} v=1$ & 6.25 & - \\
$\mathrm{AG776}$ & Cont. & $v=2+350$ & 50.0 & - & ${ }^{28} \mathrm{SiO} v=2$ & 6.25 & - \\
$\mathrm{AG776}$ & Cont. & $v=2-350$ & 50.0 & - & ${ }^{28} \mathrm{SiO} v=2$ & 6.25 & - \\
$\mathrm{AG} 575$ & Line & ${ }^{29} \mathrm{SiO} v=0$ & 6.25 & 97.7 & ${ }^{28} \mathrm{SiO} v=1$ & 6.25 & 97.7 \\
\end{tabular}

Note. - Cont. = Continuum and Line = Spectral-line mode: 64 (32) channels of 97.7 (390.6) $\mathrm{KHz}$ width (CW) within a 6.25 (12.5) $\mathrm{MHz}$ bandwidth (BW).

TABLE 3

Summary of Observational Results

\begin{tabular}{|c|c|c|c|c|c|c|c|}
\hline \multirow[t]{2}{*}{ Date } & \multirow{2}{*}{$\begin{array}{c}\text { Transitions } \\
J=1-0\end{array}$} & \multirow{2}{*}{$\begin{array}{c}\text { Synthesized Beam }(1) \\
\theta_{M}\left(^{(\prime)} \times \theta_{m}\left({ }^{\prime \prime}\right) ; \text { P.A. }\left({ }^{\circ}\right)\right.\end{array}$} & \multirow{2}{*}{$\begin{array}{c}\operatorname{RMS}^{(2)} \\
\left(\text { mJy beam }^{-1}\right)\end{array}$} & \multicolumn{2}{|c|}{ Blue Peak ${ }^{(3)}$} & \multicolumn{2}{|c|}{ Red Peak ${ }^{(3)}$} \\
\hline & & & & $\mathrm{V}\left(\mathrm{km} \mathrm{s}^{-1}\right)$ & $\mathrm{I}\left(\mathrm{Jy} \mathrm{beam}^{-1}\right)$ & $\mathrm{V}\left(\mathrm{km} \mathrm{s}^{-1}\right)$ & $\mathrm{I}\left(\mathrm{Jy} \mathrm{beam}^{-1}\right)$ \\
\hline \multirow[t]{5}{*}{ AG776 } & ${ }^{28} \mathrm{SiO} v=0$ & $0.23 \times 0.19 ; 34$ & $7-113$ & -5.8 & 10 & 13.1 & 4.9 \\
\hline & ${ }^{28} \mathrm{SiO} v=1$ & $0.23 \times 0.19 ; 33$ & $15-170$ & -4.5 & 1590 & 14.5 & 930 \\
\hline & ${ }^{28} \mathrm{SiO} v=2$ & $0.24 \times 0.15 ; 20$ & $6-40$ & -6.6 & 470 & 20.0 & 380 \\
\hline & ${ }^{29} \mathrm{SiO} v=0$ & $0.22 \times 0.19 ; 25$ & 5 & -3.7 & 0.044 & 15.5 & 0.12 \\
\hline & ${ }^{30} \mathrm{SiO} v=0$ & $0.25 \times 0.15 ; 21$ & 5 & -4.6 & 0.068 & 14.8 & 0.072 \\
\hline \multirow[t]{2}{*}{ AG575 } & ${ }^{28} \mathrm{SiO} v=1$ & $0.20 \times 0.17 ; 35$ & $11-2003$ & -3.2 & 340 & 14.5 & 210 \\
\hline & ${ }^{29} \mathrm{SiO} v=0$ & $0.20 \times 0.17 ; 7$ & $7-27$ & $\ldots$ & $<0.05$ & 16.2 & 0.34 \\
\hline \multirow[t]{2}{*}{ AG575 } & ${ }^{28} \mathrm{SiO} v=1$ & $0.061 \times 0.045 ; 53$ & $50-200$ & -3.9 & 600 & 13.8 & 400 \\
\hline & ${ }^{29} \mathrm{SiO} v=0$ & $0.059 \times 0.042 ; 45$ & 16 & $\ldots$ & $<0.07$ & 15.9 & 0.840 \\
\hline
\end{tabular}

Note. -

(1) The synthesized beam results from a robust 0 weighting.

(2) $1 \sigma$ RMS noise in a 0.7 or $2.7 \mathrm{~km} \mathrm{~s}^{-1}$ channel, based on histogram fits to the pixels over the channel image.

(3) Velocity and intensity of the emission peak on the blue and red side of the spectra.

structure of the $v=1,2$ emission using VLBA images from a monthly monitoring of Source I over several years (Matthews et al. 2007; Matthews et al. in prep.). After averaging in velocity to account for different spectral resolution, the VLBA images were convolved with the VLA beam, and the offset between the $v=1$ and $v=2$ emission was measured. We did tests using 4 to 5 different velocity ranges in the $v=1,2$ spectra at 4 to 5 different epochs from the VLBA monitoring and obtained consistent results with an offset of $\Delta \alpha=14 \pm 3$ and $\Delta \delta=0.5 \pm 3$ mas. The accuracy in the computed offsets is dominated by the variation of the structure and of the relative locations of the $v=1$ and $v=2$ maser centroids from channel to channel and from epoch to epoch, rather than by the uncertainties in measuring the locations of the $v=1,2$ emission peaks. The quoted uncertainty is then the variance of the offset values measured in several channels and at different epochs.

After applying the angular offset per $\mathrm{MHz}$ relation derived above and the offset between the $v=1$ and $v=2$ emission, we estimate that the maps of the various transitions are aligned on the sky with accuracy of 3 mas in each direction.

\subsection{Absolute astrometry}

The application of the phase solutions derived from 2minute scan averages of J0541-0541 to the maser data imposes an absolute astrometry (Section 2.2). However, because J0541-0541 was observed relatively infre- quently (every 25 minutes) compared with the timescale of tropospheric and instrumental phase variations, the absolute astrometry is poor, and further alignment is necessary. In this case, absolute positions were established by comparing positions of $\mathrm{BN}$ in our maps with the position derived by Gomez et al. (2008) from VLA observations conducted in May 2006 at $8.4 \mathrm{GHz}$, $\alpha(J 2000)=05^{h} 35^{m} 14^{s} .1099, \delta(J 2000)=-05^{\circ} 22^{\prime} 22^{\prime \prime} \cdot 741$ (positional accuracy $\sim 0$.'01) and their estimated proper

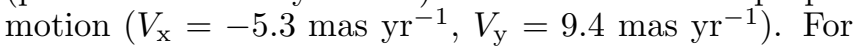
2008 February, we obtain $\alpha(J 2000)=05^{h} 35^{m} 14 . s 1093$, $\delta(J 2000)=-05^{\circ} 22^{\prime} 22^{\prime \prime} .7253$. The offset between the position of BN measured in our maps and and the propermotion-corrected position from Gomez et al. (2008) was applied to the $\mathrm{SiO}$ images, whose absolute position is then measured to an accuracy of 0. ! 01 .

\subsection{Fitting positional centroids of maser emission}

The emission in each velocity channel is unresolved in all transitions except the ${ }^{28} \mathrm{SiO} v=0$. For the latter, in order to establish the morphology and kinematics of the extended emission, we produced an intensity map integrated over the emission velocity range. For each unresolved emission component, a two-dimensional ellipsoidal Gaussian model is fitted in a $100 \times 100$ pixels area of each channel map. Since the angular extent of the maser emission in each velocity channel is smaller than the synthesized beams of the VLA in the observing configurations, spatially separated maser components at similar veloci- 
ties are blended together. Hence, the Gaussian-fitted positions actually locate the intensity-weighted centroids of $\mathrm{SiO}$ emission in each frequency channel. In the following, we will refer to these emission centroids in individual velocity channels as maser features.

The relative positional errors of maser features are limited by the SNR of the channel maps and by systematic errors in the calibration of the bandpass. In the case of weak signals (e.g., ${ }^{29} \mathrm{SiO}$ and ${ }^{30} \mathrm{SiO}$ ), the positional uncertainties are noise limited, and are given by $\delta \theta=$ $0.5 \theta_{B} / \mathrm{SNR}$, where $\theta_{B}$ is the FWHM of the synthesized beam, and SNR is the peak intensity divided by the RMS noise in a particular velocity channel. For the 2008 maps $\left(\theta_{B}=0\right.$ "' 2$)$, the relative positional uncertainties are typically $\sim 10$ mas for ${ }^{29} \mathrm{SiO}$ and ${ }^{30} \mathrm{SiO} \quad\left(\sim 50 \mathrm{mJy}\right.$ beam ${ }^{-1}$ peak and $\sim 5$ mJy beam ${ }^{-1}$ RMS), whereas for the 1999 maps $\left(\theta_{B}=\sim 50\right.$ mas $)$, the typical uncertainties are $\sim 1$ mas for ${ }^{29} \mathrm{SiO}\left(\sim 500 \mathrm{mJy}\right.$ beam ${ }^{-1}$ peak and $\left.\sim 17 \mathrm{mJy}_{\text {beam }^{-1}} \mathrm{RMS}\right)$. For the $v=1,2$ emission, the positional uncertainties are dominated by frequencydependent errors in calibrating the bandpass. From the continuum spectrum of $3 \mathrm{C} 84$, we measure a channel to channel phase noise $<1^{\circ}$, which corresponds to an uncertainty of $<0.6$ mas for a $0 .{ }^{\prime \prime} 2$ synthesized beam.

\section{RESULTS}

We imaged the ${ }^{28} \mathrm{SiO} v=0,1,2$ and the ${ }^{29} \mathrm{SiO}$ and ${ }^{30} \mathrm{SiO} v=0 J=1-0$ transitions observed in $2008\left(0{ }^{\prime \prime} 2\right.$ beam), as well as the ${ }^{28} \mathrm{SiO} v=1$ and ${ }^{29} \mathrm{SiO} v=0$ transitions observed in $2000(0 ., 2$ beam $)$ and $1999(0 . \prime 05$ beam).

In each epoch and for each transition, we produced spectra by mapping each spectral channel (0.68 or $2.7 \mathrm{~km} \mathrm{~s}^{-1}$ wide) and then summing up the flux density in each channel map. Figure 1 compares for each transition the integrated flux densities of the VLA channel maps at different epochs with the GBT single-dish spectra (observed in 2007 November 10: Goddi et al. 2009). Multiple transitions show similar double-peaked profiles and velocity extents: blueshifted emission in the velocity range from -10 to $0 \mathrm{~km} \mathrm{~s}^{-1}$, redshifted emission in the interval from 10 to $25 \mathrm{~km} \mathrm{~s}^{-1}$, and systemic emission in the range from 0 to $10 \mathrm{~km} \mathrm{~s}^{-1}$. Comparison of the GBT (2007 November) and the VLA (2008 February) spectra, shows an increase in the flux density of about $100 \%$ in the emission of $v=1$ (both blue and red components) and $v=2$ (only red component), whereas the ${ }^{28} \mathrm{SiO} v=0$ emission does not show any significant variation. Conversely, less than $\sim 30 \%$ of the single-dish emission has been detected in the VLA channel maps for ${ }^{29} \mathrm{SiO}$ and ${ }^{30} \mathrm{SiO}$. The single-dish spectral profiles of the latter two transitions appear much broader than the VLA profiles and show high-velocity wings undetected in the VLA channel maps (Fig. 1). Interestingly, the ${ }^{29} \mathrm{SiO}$ spectrum has undergone significant changes over 8-9 years (the separation between VLA epochs). In the spectra taken in 1999 and 2000, the redshifted component is 10 and 5 times stronger, respectively, than in the 2008 spectrum while no emission from the blue component is detected above a $4 \sigma \sim 50-70$ mJy noise level (Fig. 1).

The velocity integrated intensity map of ${ }^{28} \mathrm{SiO} v=$ 0 emission consists of two bright peaks offset $\sim \pm 00^{\prime \prime} 5$ from Source I (Fig. 2, left panel). The bipolar structure

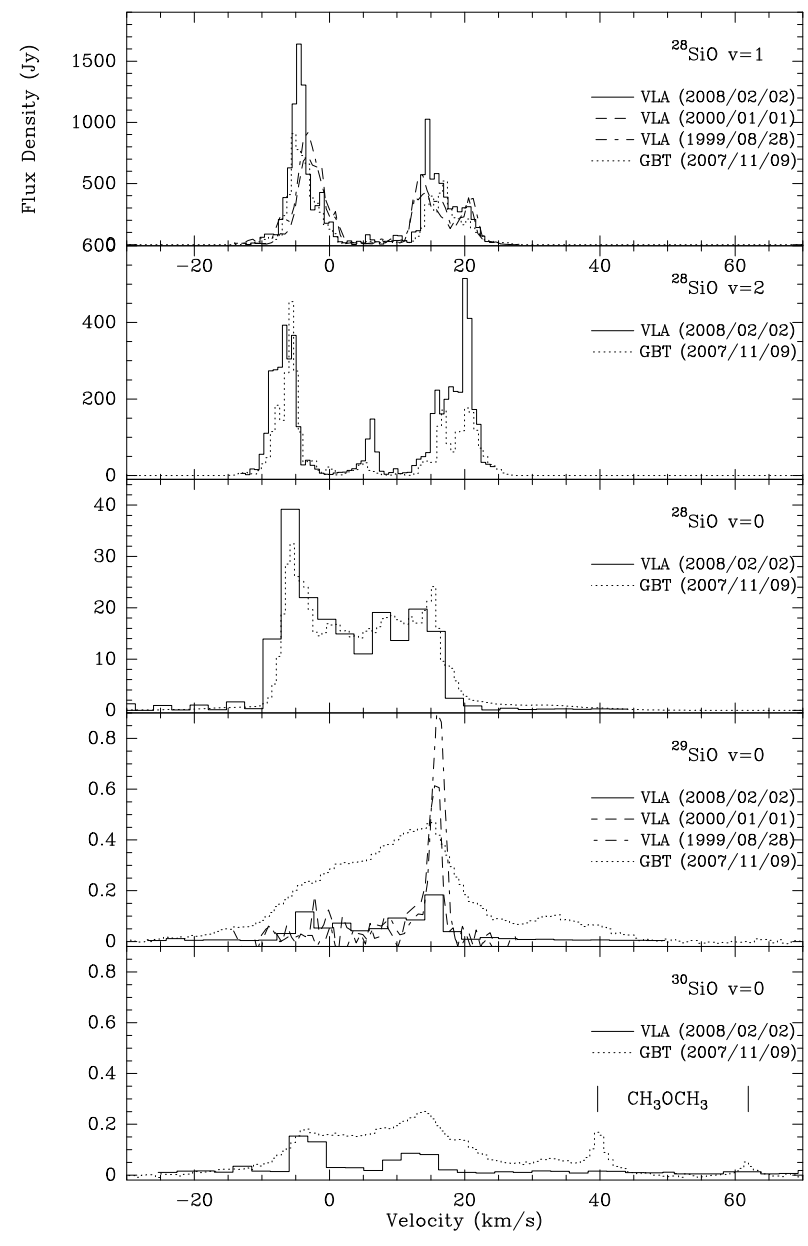

Fig. 1. - Spectra of various $\mathrm{SiO}$ transitions observed toward Orion BN/KL with the VLA during several epochs. GBT spectra from Goddi et al. (2009) are also shown for comparison. In 2008, the VLA spectral resolution was $97.7 \mathrm{kHz}\left(0.68 \mathrm{~km} \mathrm{~s}^{-1}\right)$ for the ${ }^{28} \mathrm{SiO} v=1,2 J=1-0$ lines and $390.6 \mathrm{KHz}\left(2.7 \mathrm{~km} \mathrm{~s}^{-1}\right)$ for the ${ }^{28} \mathrm{SiO},{ }^{29} \mathrm{SiO}$, and ${ }^{30} \mathrm{SiO} v=0 J=1-0$ lines. In 1999 and $2000,{ }^{28} \mathrm{SiO} v=1$ and ${ }^{29} \mathrm{SiO} v=0$ were observed with a $0.68 \mathrm{~km} \mathrm{~s}^{-1}$ resolution. The large differences between GBT and VLA $v=1,2$ spectra is likely to be due to the time separation of observations and the strongly maser-nature and compactness of the emission. In the case of the isotopologues, most probably the observed difference is due to an extended, thermal component detected by the single-dish but resolved out by the interferometer. Note that the same flux density scale is adopted for ${ }^{29} \mathrm{SiO}$ and ${ }^{30} \mathrm{SiO}$.

extends over $\sim 700 \mathrm{AU}$ along a NE-SW direction and is similar to previous BIMA images of the $J=2-1$ line (Wright et al. 1995). Both lobes emit in a similar velocity interval, from -9 to $16 \mathrm{~km} \mathrm{~s}^{-1}$.

The emission centroids from ${ }^{28} \mathrm{SiO} v=1,2$ and ${ }^{29} \mathrm{SiO}$ and ${ }^{30} \mathrm{SiO} v=0$ are clearly located at the center of the ${ }^{28} \mathrm{SiO} v=0$ bipolar structure (Fig. 2, left panel) and are distributed in a region of size $0 . \prime 2$ around Source I (Fig. 2, right panel). They show generally similar spatial and velocity distributions and can be divided into three groups: blue features detected southeast (SE) of Source I include emission with line-of-sight (L.O.S.) velocities blueshifted with respect to the systemic LSR velocity of the region $\left(5 \mathrm{~km} \mathrm{~s}^{-1}\right)$, red features located to the north-west (NW) have emission with redshifted L.O.S. velocities, and systemic features, which are weaker and located in between the two other groups, are com- 
paratively close to the systemic velocity. However, some differences can be noted among various transitions. Compared to $v=1$, the ${ }^{28} \mathrm{SiO} v=2$ emission appears located in a slightly inner layer, closer to Source I, even if there is a significant overlap. In addition, the systemic features appear stronger (relative to blue and red peaks) for $v=2$ than for $v=1$. This is consistent with the findings of higher-resolution imaging of the ${ }^{28} \mathrm{SiO} v=1,2$ emission with the VLBA (Matthews et al. 2007; Matthews et al. in prep.).

As far as the isotopologues are concerned, although correspondence among ground-state isotopologue and vibrationally excited emission distributions is generally good, a one-to-one correspondence in positions and L.O.S. velocities in features among different transitions is clearly absent. In addition, the brightest features from the main species and isotopologues are not co-spatial (with the exception of the ${ }^{29} \mathrm{SiO}$ red features in the NW area). Finally, while most of the blue and systemic ${ }^{29} \mathrm{SiO}$ features also have corresponding ${ }^{30} \mathrm{SiO}$ features, this is not necessarily true for the red features.

We caution that, owing to low angular and spectral resolution, blending may hamper detailed comparisons of emission centroid positions. For instance, higher angular $\left(0\right.$.'05 beam) and spectral $\left(0.68 \mathrm{~km} \mathrm{~s}^{-1}\right)$ resolution imaging with the VLA reveals that the ${ }^{28} \mathrm{SiO} v=1$ maser emission does not arise from two (blue and red) arcs (as shown in Fig. 2), but resolves into an "X"shaped pattern, consisting of four "arms", extending towards north $(\mathrm{N})$, west $(\mathrm{W})$, south $(\mathrm{S})$, and east $(\mathrm{E})$, plus a bridge of emission joining the $\mathrm{S}$ and $\mathrm{W}$ arms of the "X" (Figure 31). The X-shaped structure is consistent with sub-milliarcsecond resolution VLBA imaging (Greenhill et al. 1998; Doeleman et al. 2004; Matthews et al. in prep.). In the high-resolution VLA map, most of the red features arise from equally bright components in the $\mathrm{N}$ and $\mathrm{W}$ arms, the blue features from components in the $\mathrm{S}$ and $\mathrm{E}$ arms, and the systemic features from the bridge (Figure 3). As a consequence, with a 0.2 angular resolution and $2.7 \mathrm{~km} \mathrm{~s}^{-1}$ spectral resolution, centroids of both red and blue features fall between $\mathrm{N}$ and $\mathrm{W}$ arms and $\mathrm{S}$ and $\mathrm{E}$ arms, respectively, resulting in the two (red and blue) arcs seen in Fig. 2. Similar arc morphologies have been previously seen in low-resolution imaging at $7 \mathrm{~mm}$ with the VLA (0.'2 beam - Menten \& Reid 1995) and at $3 \mathrm{~mm}$ with BIMA (0.5 beam - Wright et al. 1995) and PdBI (2" beam - Baudry et al. 1998).

The high resolution VLA image allows accurate localization of the ${ }^{29} \mathrm{SiO}$ emission towards the $\mathrm{N}$ arm of the $\mathrm{X}$ traced by the $v=1$ emission (Fig. 3), which invites speculation that the presence of isotopologue emission within the arcs on either side of Source I (Fig. 2), when observed at lower resolution, is indicative of an origin in the four arms. Nonetheless, detailed comparison requires caution in light of the apparent 2-10 AU offset between ${ }^{29} \mathrm{SiO}$ and ${ }^{28} \mathrm{SiO}$ features of similar velocity (Fig. 33).

\section{DISCUSSION}

\subsection{Gas kinematics around Source I}

The NE/SW-oriented bipolar structure traced by the ${ }^{28} \mathrm{SiO}$ vibrational ground state has been previously interpreted either as a rotating disk, based on maps of the $J=$ $2-1$ emission at $3 \mathrm{~mm}$ wavelength (Wright et al. 1995), or as a bipolar outflow, based on the distributions of hotspots of $J=1-0$ emission at $7 \mathrm{~mm}$ Greenhill et al. 2004a) and the distribution of the $J=8-7$ emission at $0.9 \mathrm{~mm}$ (Beuther et al. 2005). The data presented here are consistent with the outflow hypothesis, where we cite: (i) a fully developed bipolar geometry when imaging detects both hotspots (Greenhill et al. 2004a) and extended structure (this work); (ii) lobes that exhibit similar mixes of blue and redshifted components, indicative of outflow close to the plane of the sky rather than rotation; and (iii) the putative outflow in the vicinity of Source I is aligned with the principle axis of the " $18 \mathrm{~km} \mathrm{~s}^{-1}$ " outflow in the BN/KL region (Genzel et al. 1981), which is suggestive of Source I as a possible contributor.

The X-shaped distribution of vibrationally excited ${ }^{28} \mathrm{SiO}$ emission has been interpreted as tracing either a biconical outflow with a SE-NW axis (Greenhill et al. 1998; Doeleman et al. 1999), funnel-like flows arising from the surface of a disk with a NE-SW rotation axis (Greenhill et al. 2004a), or the interface between infalling and outflowing material Cunningham et al. 2005).

Emission from both vibrationally excited states of the main species and the ground state of the isotopologues traces the innermost $(\lesssim 100 \mathrm{AU})$ circumstellar gas around Source I (Fig. 2 and [3). Notwithstanding similarities, it is not however possible to make an exact positionvelocity-intensity pairing of the ${ }^{28} \mathrm{SiO} v=1,2$ and ${ }^{29} \mathrm{SiO} /{ }^{30} \mathrm{SiO} v=0$ features (Sect. [3). Hence, it remains possible that the main and isotopic species might be excited in somewhat different volumes and in principle might require slightly different physical conditions (see Sections 4.3). A corresponding uncertainty in interpretation has been raised by Baudry et al. (1998) to explain differences in position between the ${ }^{28} \mathrm{SiO} v=1$ and ${ }^{29} \mathrm{SiO} v=0 \mathrm{~J}=2-1$ emission in $3 \mathrm{~mm}$ wavelength PdBI images.

Previous imaging of thermal emission from ${ }^{28} \mathrm{SiO}$ and ${ }^{30} \mathrm{SiO}(v=0 J=8-7)$ in the submm-wave band with the SMA has shown that both ${ }^{28} \mathrm{SiO}$ and ${ }^{30} \mathrm{SiO}$ emissions subtend similar angles on the sky (size $1000 \mathrm{AU}$ ), are elongated NE-SW, and cover about the same velocity range (Beuther et al. 2005). The natural inference is that both species may trace inner reaches of the NE-SW outflow. Our VLA maps, however, do not show any indication of isotopic emission within the NE-SW outflow traced by ${ }^{28} \mathrm{SiO} v=0$ emission. The actual spatial structure of the ${ }^{29} \mathrm{SiO}$ and ${ }^{30} \mathrm{SiO} v=0 \mathrm{~J}=1-0$ emission might be more complex and extended than illustrated in Figures 2 and 3. The large difference in flux between the single-dish and the interferometer spectra (Fig. 1), may be in fact ascribed to an extended, low-surface brightness component, detected by the GBT but filtered out by the VLA. Indeed, in our VLA maps we are insensitive to emission with an integrated flux density $\lesssim 100 \mathrm{mJy}$ and that extends over $\gtrsim 1^{\prime \prime}$ (note that the line wings observed in the GBT spectra for ${ }^{29} \mathrm{SiO}$ and ${ }^{30} \mathrm{SiO}$ are $\sim 100 \mathrm{mJy})$. Hence, the ${ }^{29} \mathrm{SiO}$ and ${ }^{30} \mathrm{SiO}$ emission imaged with the VLA traces only the highest brightness regions in the proximity of Source I. With the present VLA data, we cannot establish the location of the moderately high-velocity component of the protostellar outflow from Source I, as traced by the $v=0$ wing emission observed with the GBT. 


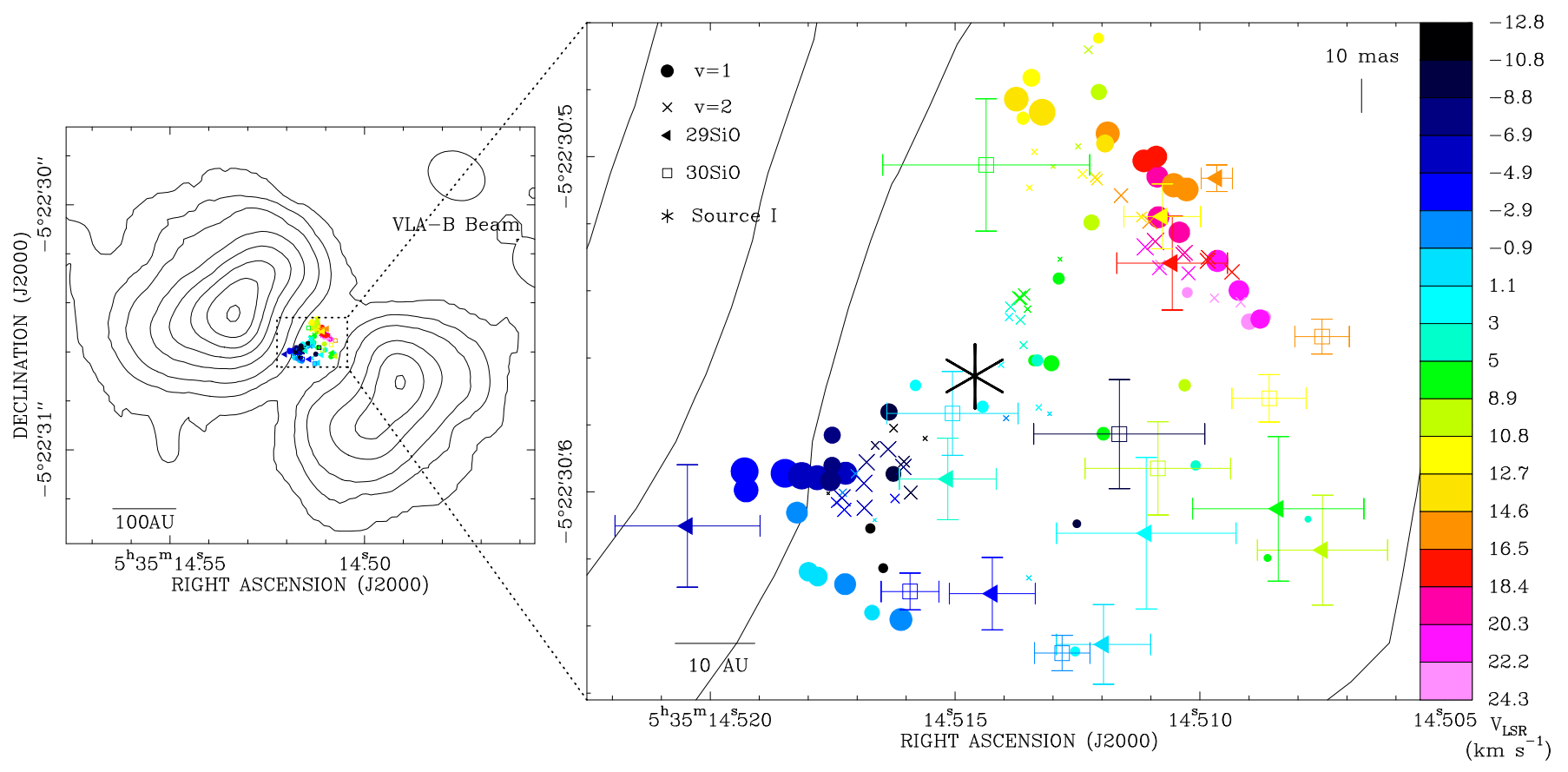

FIG. 2.- Distribution of isotopic $\mathrm{SiO}$ maser emission in Orion BN/KL observed on 2008 February 2. (Left) Emission map of ${ }^{28} \mathrm{SiO} v=$ $0 J=1-0$ is integrated from -11 to $21 \mathrm{~km} \mathrm{~s}^{-1}$. The contour levels are 1, 5, 10, 20,30, 40,50,60 Jy beam ${ }^{-1} \mathrm{~km} \mathrm{~s}^{-1}$. The map shows a bow tie shape similar to that observed previously at $3 \mathrm{~mm}$ wavelength with BIMA (Wright et al. 1995). Source I is located at the center of the ${ }^{28} \mathrm{SiO} v=0$ flow, where the emission from other isotopologues and vibrational states also lie. The beam $\left(0 !^{\prime \prime} 22 \times 00^{\prime \prime} 19, \mathrm{P} . \mathrm{A}\right.$. $\left.25^{\circ}\right)$ is shown on the right upper corner. (Right) Emission centroids of ${ }^{28} \mathrm{SiO} v=1$ (filled circles) and $v=2$ (crosses), ${ }^{29} \mathrm{SiO}$ (filled triangles) and ${ }^{30} \mathrm{SiO} v=0$ (open squares) as a function of velocity superimposed on the velocity integrated intensity map of ${ }^{28} \mathrm{SiO} v=0$ (contours). Color denotes L.O.S. velocity (color scale on the right-hand side). The sizes of circles and crosses scale logarithmically with the peak intensity of the $v=1,2$ emission. For ${ }^{29} \mathrm{SiO}$ and ${ }^{30} \mathrm{SiO}$ the position uncertainties reflect formal errors in model fits. The adopted absolute position of Source I is $\alpha(J 2000)=05^{h} 35^{m} 14^{s} .5146 \pm 0.0007, \delta(J 2000)=-05^{\circ} 22^{\prime} 30^{\prime \prime} 5655 \pm 0^{\prime \prime} 01$, after correction for proper motions (Gomez et al. 2008); the size of the star indicates uncertainty. The linear and angular spatial scales are given in the lower left and upper right corners of the panel, respectively. The relative alignment between pairs ${ }^{28} \mathrm{SiO} v=1$ and ${ }^{28} \mathrm{SiO} /{ }^{29} \mathrm{SiO} v=0$ or ${ }^{28} \mathrm{SiO} v=2$ and ${ }^{30} \mathrm{SiO} v=0$ is accurate to $\sim 3$ mas. The uncertainty in the absolute position is $\sim 10$ mas.

\subsection{Nature of the isotopologue emission}

The ${ }^{29} \mathrm{SiO}$ and ${ }^{30} \mathrm{SiO} v=0 J=1-0$ emissions have been imaged at sub-arcsecond resolution for the first time. Chandler \& de Pree (1995) showed that the ${ }^{28} \mathrm{SiO} v=0 J=1-0$ emission in Orion $\mathrm{BN} / \mathrm{KL}$ is part thermal and part maser $\left(T_{b} \sim 10^{5} \mathrm{~K}\right)$. Baudry et al. (1998) reached the same conclusion for the ${ }^{29} \mathrm{SiO} v=$ $0 \mathrm{~J}=2-1$ emission $\left(T_{b} \sim 10^{3} \mathrm{~K}\right)$.

On one hand, the broad profiles and the large flux densities at systemic velocities in the ground-state emission as compared to the vibrationally excited states (Fig. 1) clearly indicates thermal emission from all species. On the other hand, the high flux density measured for the strongest features in the interferometric maps suggests, similarly to ${ }^{28} \mathrm{SiO}$, non-thermal emission for ${ }^{29} \mathrm{SiO}$ and ${ }^{30} \mathrm{SiO} v=0 \mathrm{~J}=1-0$ as well. Although our observations do not resolve the emission in individual velocity channels (and hence we do not know the actual size of individual features), we may use the synthesized beamwidth and the observed peak flux density at different angular resolutions to estimate a minimum brightness temperature. For ${ }^{29} \mathrm{SiO}$, the peak intensity measured in the $16 \mathrm{~km} \mathrm{~s}^{-1}$ channel map with a 0.2 resolution is $0.12 \mathrm{Jy} \mathrm{beam}^{-1}$, which corresponds to a line brightness temperature of $>1900 \mathrm{~K}$. In the 1999 observations, with an angular resolution of 0. " 05 , the same velocity component has a peak intensity of $\sim 0.84 \mathrm{Jy} /$ beam, corresponding to a brightness temperature of $>2 \times 10^{5} \mathrm{~K}$. Hence, the bright feature at $16 \mathrm{~km} \mathrm{~s}^{-1}$ is undoubtedly a maser.
Temporal variations in the ${ }^{29} \mathrm{SiO}$ line profile (Fig. 1) further support non-thermal processes in the excitation of ${ }^{29} \mathrm{SiO}$.

The ${ }^{30} \mathrm{SiO}$ emission is likely maser in nature as well. For the strongest ${ }^{30} \mathrm{SiO}$ emission in the $-4.8,-1.8$, and $14.8 \mathrm{~km} \mathrm{~s}^{-1}$ channels, we infer a brightness temperature of $>1200 \mathrm{~K}$ (for a 0.2 beam), which is comparable to the value inferred for ${ }^{29} \mathrm{SiO}$ from maps with the same resolution. The estimated value is well above the kinetic temperature observed even in the nearby hot core (150-300 K) (e.g., Genzel \& Stutzki 1989), but it could be plausible in principle in shocked gas, where the $\mathrm{SiO}$ molecule is believed to be produced and excited. In particular, the $\mathrm{SiO}$ molecule is produced in C-type shocks by the injection into the gas-phase of $\mathrm{Si}$ by grain sputtering and/or grain-grain collisions, followed by gas-phase reactions with $\mathrm{O} / \mathrm{O}_{2}$ (Schilke et al. 1997; Caselli et al. 1997). At typical speeds of C-type shocks $\left(10-50 \mathrm{~km} \mathrm{~s}^{-1}\right)$, the temperature of the post-shocked gas rises up to $1000 \mathrm{~K}$ and then progressively decreases to its pre-shock values. Multi-line $\mathrm{SiO}$ observations in protostellar jets give kinetic temperatures $<1000 \mathrm{~K}$ (typical values in the range 100-500 K: Nisini et al. 2007; Cabrit et al. 2007). Hence, if the emission were thermal, the inferred brightness temperature would be only marginally consistent with the temperatures that could be achieved via C-type shocks and/or with typically observed kinetic temperatures in $\mathrm{SiO}$ protostellar jets. Based on similarities (e.g., compactness) with all other masers lines $\left({ }^{28} \mathrm{SiO} v=1,2\right.$ and 


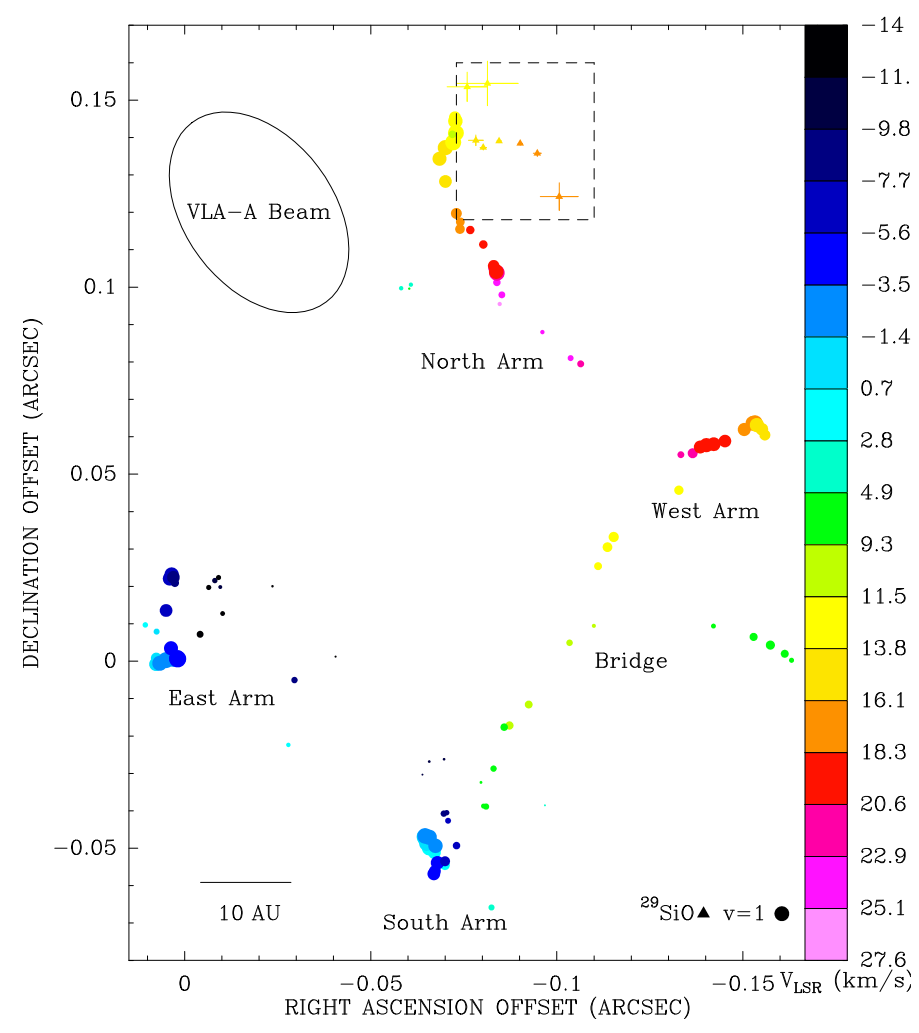

Fig. 3.- Centroids of ${ }^{28} \mathrm{SiO} v=1$ (filled circles) and ${ }^{29} \mathrm{SiO} v=0$ (filled triangles with error bars) compact emission observed on 1999 August 28. The ${ }^{28} \mathrm{SiO} v=1$ emission distribution traces a 4-arm structure, redshifted in the north and west arms and blueshifted in the south and east arms. Note also a weaker bridge of emission connecting west and south arms. The ${ }^{29} \mathrm{SiO}$ emission is concentrated towards the north arm (dashed rectangular area). The ellipse on the top left indicates the synthesized beam $\left(0 !^{\prime \prime} 06\right.$ $\times 0 !^{\prime \prime} 04 ;$ P.A. $\left.53^{\circ}\right)$. Since the beam size is comparable to the arm extensions, the $v=1$ emission from each arm is not resolved in the map, and the specific structure within each arm may be artifactual owing to spatial blending (compare with the VLBA images from Greenhill et al. 1998). Note that the same physical scale is used in both Fig. 2 and Fig. 3

${ }^{29} \mathrm{SiO} v=0$ ), the ${ }^{30} \mathrm{SiO}$ emission is more likely to be a maser.

For some of the weak ${ }^{29} \mathrm{SiO}$ and ${ }^{30} \mathrm{SiO}$ features we obtain brightness temperatures in the range $260-400 \mathrm{~K}$, indicating that the isotopic $v=0$ emission imaged with the VLA is probably part thermal and part maser as for the ${ }^{28} \mathrm{SiO} v=0$ emission. The difference between the singledish and interferometric spectral profiles indicates that the VLA in B-configuration does not have the brightness temperature sensitivity (RMS noise $T_{b} \sim 80 \mathrm{~K}$ ) to detect the weaker and extended thermal component (Sect.4.1).

Based on our single-dish spectra and interferometric images, we conclude that the $v=0$ emission from all species is a combination of thermal and maser components.

\subsection{Physical conditions from a radiative transfer modeling}

The ground-state isotopologue and the vibrationally excited state emissions appear to arise from gas within $\sim 100$ AU of Source I, but possibly at somewhat different locations within the volume. We have undertaken preliminary radiative transfer calculations to assess whether putative offsets might be due to excitation requirements for the different transitions.
We used a radiative transfer code based on the Large Velocity Gradient (LVG) model of Doel et al. (1995), that includes both collisional and radiative pumping but does not treat effects of maser saturation or line overlap. We carried out model calculations using 600 energy levels $(v=0-4 ; J=0-39$ for each isotopic species). The main input parameters for the LVG model are reported in Table 4. The model background continuum assumes a blackbody function of $T_{*}=10^{4} \mathrm{~K}$. The radiation field is reduced by a geometric dilution factor, $W=1 / 4\left(R_{\mathrm{rad}}^{2} / R_{\mathrm{mas}}^{2}\right)$, where $R_{\mathrm{rad}}$ is the radius of the radiative source and $R_{\text {mas }}$ is the distance to the maser region. The lower limit of $R_{\text {rad }}$ corresponds to a B star photospheric radius $\left(7 \times R_{\odot}\right)$ (Zombeck 2007) and the upper limit roughly approximates the radio continuum size (Reid et al. 2007). We do not take into account emission from dust around Source I as dust is likely to be sublimated at radii $<100$ AU, where highdensity $\mathrm{SiO}$ in gas phase is present. A velocity gradient $d \mathrm{~V} / d r=0.5 \mathrm{~km} \mathrm{~s}^{-1} \mathrm{AU}^{-1}$ is assumed based on the velocity dispersion measured across the arms of the $\mathrm{X}$ in the $v=1$ emission (Fig. 3). We adopt the following relative (approximately solar) abundances: $\left[\mathrm{SiO} / \mathrm{H}_{2}\right]=$ $10^{-4},\left[{ }^{28} \mathrm{SiO}\right] /\left[{ }^{29} \mathrm{SiO}\right] \sim 19.5$, and $\left[{ }^{28} \mathrm{SiO}\right] /\left[{ }^{30} \mathrm{SiO}\right] \sim 30.5$ (Penzias 1981)). The Einstein coefficients are calculated from the data of Tipping \& Chackerian (1981), while the collisional rate coefficients are taken from Bieniek \& Green (1983a, b) .

For simplicity, we assume that the velocity field is spherically-symmetric at any point in the medium, i.e. the logarithmic velocity gradient $d(\ln \mathrm{V}) / d r=1.0$. The photon escape probability is then given by $\beta=$ $\left(1-e^{-\tau_{s}}\right) / \tau_{s}$, where the Sobolev optical depth is $\tau_{s}=$ $\left(c^{3} / 8 \pi \nu_{u l}^{3}\right) A_{u l}\left[n_{u}-n_{l} g_{u} / g_{l}\right] /(d \mathrm{~V} / d r)$, where $d \mathrm{~V} / d r$ is the velocity gradient and $n_{u}$ and $n_{l}$ are populations per sublevel. Calculated population inversions are represented in terms of the gain coefficient $\gamma=\lambda^{2} \Delta n / 8 \pi t_{s}$, where $\Delta n$ is the population inversion and $t_{s}$ is the radiative lifetime of the transition.

The physical conditions required for the excitation of different maser species are summarized in Table 5. The ${ }^{28} \mathrm{SiO} v=0 \mathrm{~J}=1-0$ transition is inverted at relatively low density $\left(\mathrm{N}_{\mathrm{H}_{2}}<10^{7} \mathrm{~cm}^{-3}\right)$, and it is never inverted in a strong, hot radiation field $(W<0.001)$, consistent with an inner radius of 200-1000 AU (Sect. 3). It occurs at a wide range of kinetic temperatures but it is optimized at $T_{k}<1200 \mathrm{~K}$.

The ${ }^{28} \mathrm{SiO} v=1,2 J=1-0$ transitions are never inverted for $\mathrm{N}_{\mathrm{H}_{2}}<10^{7} \mathrm{~cm}^{-3}$ and they favor densities of $10^{8}-10^{10} \mathrm{~cm}^{-3}(v=1)$ and $10^{9}-10^{11} \mathrm{~cm}^{-3}$ $(v=2)$. These results explain the spatial separation between the ground $(\gtrsim 100 \mathrm{AU})$ and excited vibrational states $(\lesssim 100 \mathrm{AU})$. The $v=2$ transition is optimized at higher temperatures $\left(T_{k} \sim 2000 \mathrm{~K}\right)$ and it is more strongly inverted in a strong, hot radiation field than the $v=1$ transition, which is apparently quenched for $W>0.01$. This is in agreement with the finding that $v=2$ masers tend to lie closer to the star than $v=1$ masers, although there is substantial spatial overlap (Sect. 3).

The ${ }^{29} \mathrm{SiO}$ and ${ }^{30} \mathrm{SiO} v=0 J=1-0$ transitions are inverted across a broad range of parameter space. Their emission is optimized for conditions similar to ${ }^{28} \mathrm{SiO} v=$ $1,2\left(\mathrm{~N}_{\mathrm{H}_{2}}=10^{8}-10^{11} \mathrm{~cm}^{-3}\right.$ and $\left.T_{k}=1000-2000 \mathrm{~K}\right)$, 
TABLE 4

INPUT PARAMETERS IN THE LVG MODEL.

\begin{tabular}{ccccccccc}
\hline \hline $\begin{array}{l}\mathrm{T}_{*}^{(1)} \\
(\mathrm{K})\end{array}$ & $\begin{array}{c}\mathrm{R}_{\mathrm{rad}}^{(2)} \\
(\mathrm{AU})\end{array}$ & $\begin{array}{c}\mathrm{R}_{\mathrm{mas}}^{(3)} \\
(\mathrm{AU})\end{array}$ & $\mathrm{W}^{(4)}$ & $\begin{array}{c}\mathrm{N}_{\mathrm{H}_{2}} \\
\left(\mathrm{~cm}^{-3}\right)\end{array}$ & $\begin{array}{c}\mathrm{T}_{k} \\
(\mathrm{~K})\end{array}$ & $\chi\left[\mathrm{SiO} / \mathrm{H}_{2}\right]^{(5)}$ & ${ }^{28} \mathrm{SiO}^{29} \mathrm{SiO}^{30} \mathrm{SiO}^{(5)}$ & $\begin{array}{c}d \mathrm{~V} / d r^{(6)} \\
\left(\mathrm{km} \mathrm{s}^{-1} \mathrm{AU}^{-1}\right)\end{array}$ \\
\hline $10^{4}$ & $0.04-5$ & $10-1000$ & $10^{-1}-10^{-8}$ & $10^{4}-10^{12}$ & $600-2400$ & $10^{-4}$ & $1: 1 / 19.5: 1 / 30.5$ & 0.5 \\
\hline
\end{tabular}

Note. -

(1) $T_{*}$ is the blackbody temperature of the radiative field.

(2) $R_{\text {rad }}$ is the radius of the radiative source.

(3) $R_{\text {mas }}$ is the distance to the maser region.

(4) $\mathrm{W}$ is the radiation geometric dilution factor.

(5) $\chi\left[\mathrm{SiO} / \mathrm{H}_{2}\right]$ and ${ }^{28} \mathrm{SiO}:{ }^{29} \mathrm{SiO}:{ }^{30} \mathrm{SiO}$ are the (solar) fractional abundances of $\mathrm{SiO}$ and isotopologues.

(6) $d \mathrm{~V} / d r$ is the velocity gradient as measured by the velocity dispersion of $v \mathrm{Si} O$ emissionsic emission arises from compact compoTABLE 5

Optimal LVG EXCITATION CONDITIONS OF SIO MASERS.

\begin{tabular}{|c|c|c|c|}
\hline Species & $\mathrm{W}$ & $\begin{array}{c}\mathrm{N}_{\mathrm{H}_{2}} \\
\left(\mathrm{~cm}^{-3}\right)\end{array}$ & $\begin{array}{l}\mathrm{T}_{k} \\
(\mathrm{~K})\end{array}$ \\
\hline${ }^{28} \mathrm{SiO} v=0$ & 0.0001 & $<10^{7}$ & $<1200$ \\
\hline${ }^{28} \mathrm{SiO} v=1$ & $<0.01$ & $10^{8}-10^{10}$ & $>1500$ \\
\hline${ }^{28} \mathrm{SiO} v=2$ & 0.01 & $10^{9}-10^{11}$ & $>2000$ \\
\hline${ }^{29} \mathrm{SiO} v=0$ & 0.01 & $10^{8}-10^{11}$ & $>1500$ \\
\hline${ }^{30} \mathrm{SiO} v=0$ & 0.01 & $10^{8}-10^{11}$ & $>1500$ \\
\hline
\end{tabular}

but can also occur more weakly under conditions conducive to ${ }^{28} \mathrm{SiO} v=0$ inversion $\left(\mathrm{N}_{\mathrm{H}_{2}}<10^{7} \mathrm{~cm}^{-3}\right)$. These elements are in agreement with our finding that strong isotopic maser emission is excited within $100 \mathrm{AU}$ from Source I as is ${ }^{28} \mathrm{SiO} v=1,2$ maser emission, while extended weak isotopic emission may occur at larger distances from Source I in more extended structures, such as along the NE-SW outflow traced by the ${ }^{28} \mathrm{SiO} v=0$ emission (Sect. 4.1).

In conclusion, our LVG radiative pumping model explains the bulk $\mathrm{SiO}$ maser emission characteristics of the five maser transitions observed toward Orion $\mathrm{BN} / \mathrm{KL}$. However, although the large-scale spatial and velocity distribution is similar for ${ }^{28} \mathrm{SiO} v=1,2$ and ${ }^{29} \mathrm{SiO} /{ }^{30} \mathrm{SiO} v=0$ maser centroids, our data indicate differences in the small-scale spatial distributions and a certain degree of anticorrelation in intensity. In order to take into account these effects, future work would need to incorporate non-local radiative transfer and possibility of line overlap among the isotopologues in the modeling of the excitation of $\mathrm{SiO}$ masers (Gonzalez-Alfonso \& Cernicharo 1997; Herpin \& Baudry 2000).

\section{SUMMARY}

We have used the VLA at $7 \mathrm{~mm}$ wavelength to image five rotational transitions from three $\mathrm{SiO}$ isotopologues towards Orion BN/KL: ${ }^{28} \mathrm{SiO} v=0,1,2$ and ${ }^{29} \mathrm{SiO}$ and ${ }^{30} \mathrm{SiO} v=0(J=1-0)$. We have imaged for the first time the ${ }^{29} \mathrm{SiO}$ and ${ }^{30} \mathrm{SiO} v=0$ maser emission with an angular resolution $\lesssim 0$ !' 2 .

The main findings of this paper are listed below.

- ${ }^{28} \mathrm{SiO} v=0$ maser emission traces a bipolar outflow driven by Source I extended over $\sim 700$ AU along a NE-SW axis. nents with a spatial and velocity distribution generally similar to the ${ }^{28} \mathrm{SiO}$ vibrationally excited emission and traces material within $100 \mathrm{AU}$ of Source I.

- With resolution of $0{ }^{\prime \prime} 2, \sim 70 \%$ of the isotopic emission appears to be resolved. The missing flux may arise in the outflow traced at larger radii by ${ }^{28} \mathrm{SiO} v=0$ emission.

- LVG radiative transfer calculations predict similar temperatures and densities for the optimum maser excitation of both ${ }^{29} \mathrm{SiO} /{ }^{30} \mathrm{SiO} v=0$ and ${ }^{28} \mathrm{SiO} v=1,2$ masers $\left(T=1000-2000 \mathrm{~K}, n_{\mathrm{H}_{2}} \sim\right.$ $\left.10^{10 \pm 1} \mathrm{~cm}^{-3}\right)$, significantly higher than what is required for ${ }^{28} \mathrm{SiO} v=0\left(T \sim 600-1200 \mathrm{~K}, n_{\mathrm{H}_{2}} \sim\right.$ $\left.10^{6 \pm 1} \mathrm{~cm}^{-3}\right)$. However, weak emission from the isotopologues cannot be excluded at the lower temperatures and densities that support ${ }^{28} \mathrm{SiO} v=0$.

- Although the emission distributions for different isotopologues are generally similar (e.g., in radius), the peaks do not appear to overlap in detail (although blending effects may contribute to the apparent offsets owing to the coarse interferometer beam).

The apparent positional offset of maser features from different transitions and/or isotopologues suggests that non-local line overlaps should be included in the modeling of the excitation of $\mathrm{SiO}$ (isotopic) masers. Follow up modeling will use a non-local spherical geometry radiative transfer code with line overlap. Higherresolution and higher sensitivity simultaneous observations of multiple maser lines/species with future facilities like EVLA and ALMA will be very important to make progress in the understanding of the gas dynamics around Source I and to better constrain physical conditions around Source I on small-scales.

The data presented here were obtained under the VLA programs AG575 and AG776. This material is based upon work supported by the National Science Foundation under Grant No. NSF AST 0507478. 
Beuther, H., et al. 2005, ApJ, 632, 355

Bieniek, R. J., \& Green, S. 1983a, ApJ, 265, L29

Bieniek, R. J., \& Green, S. 1983b, ApJ, 270, L101

Buhl, D., Snyder, L. E., Lovas, F. J., \& Johnson, D. R. 1974, ApJ, 192, L97

Buhl, D., Snyder, L. E., Lovas, F. J., \& Johnson, D. R. 1975, ApJ, 201, L29

Cabrit, S., Codella, C., Gueth, F., Nisini, B., Gusdorf, A., Dougados, C., \& Bacciotti, F. 2007, A\&A, 468, L29

Caselli, P., Hartquist, T. W., \& Havnes, O. 1997, A\&A, 322, 296

Chandler, C. J., \& de Pree, C. G. 1995, ApJ, 455, L67

Cho, S.-H., Chung, H.-S., Kim, H.-R., Kim, H.-G., \& Roh, D.-G. 1999, AJ, 117, 1485

Cho, S.-H., Kim, H.-G., Park, Y.-S., Choi, C.-H., \& Ukita, N. 2005, ApJ, 622, 390

Churchwell, E., Wood, D. O. S., Felli, M., \& Massi, M. 1987, ApJ, 321,516

Cunningham, A., Frank, A., \& Hartmann, L. 2005, ApJ, 631, 1010

Doel, R. C., Gray, M. D., Humphreys, E. M. L., Braithwaite, M. F., \& Field, D. 1995, A\&A, 302, 797

Doeleman, S. S., Lonsdale, C. J., \& Pelkey, S. 1999, ApJ, 510, L55

Doeleman, S. S., Lonsdale, C. J., Kondratko, P. T., \& Predmore, C. R. 2004, ApJ, 607, 361

Genzel, R., Downes, D., Pankonin, V., Baars, J. W. M., Schwartz, P. R., \& Spencer, J. H. 1980, ApJ, 239, 519

Genzel, R., Reid, M. J., Moran, J. M., \& Downes, D. 1981, ApJ, 244,884

Genzel, R., \& Stutzki, J. 1989, ARA\&A, 27, 41

Gezari, D. Y. 1992, ApJ, 396, L43

Goddi, C., Greenhill, L. J., Humphreys, E. M. L., Matthews, L. D., Tan, J. C., \& Chandler, C. J. 2009, ApJ, 691, 1254

Gomez, L., Rodriguez, L. F., Loinard, L., Lizano, S., Allen, C., Poveda, A., \& Menten, K. M. 2008, ArXiv e-prints, 805, arXiv:0805.3650

Gonzalez-Alfonso, E., Alcolea, J., \& Cernicharo, J. 1996, A\&A, 313, L13

Gonzalez-Alfonso, E., \& Cernicharo, J. 1997, A\&A, 322, 938

Greenhill, L. J., Gwinn, C. R., Schwartz, C., Moran, J. M., \& Diamond, P. J. 1998, Nature, 396, 650

Greenhill, L. J., Reid, M. J., Chandler, C. J., Diamond, P. J., \& Elitzur, M. 2004a, Star Formation at High Angular Resolution, 221, 155

Greenhill, L. J., Gezari, D. Y., Danchi, W. C., Najita, J., Monnier, J. D., \& Tuthill, P. G. 2004b, ApJ, 605, L57
Hasegawa, T., Morita, K., Okumura, S., Kaifu, N., Suzuki, H., Ohishi, M., Hayashi, M., \& Ukita, N. 1986, Masers, Molecules, and Mass Outflows in Star Formation Regions, 275

Herpin, F., \& Baudry, A. 2000, A\&A, 359, 1117

Lovas, F. J., Johnson, D. R., Buhl, D., \& Snyder, L. E. 1976, ApJ, 209,770

Matthews, L. D., Goddi, C., Greenhill, L. J., Chandler, C. J., Reid, M. J., \& Humphreys, E. M. L. 2007, IAU Symposium, 242, 130 Menten, K. M., \& Reid, M. J. 1995, ApJ, 445, L157

Menten, K. M., Reid, M. J., Forbrich, J., \& Brunthaler, A. 2007, A\&A, 474, 515

Nisini, B., Codella, C., Giannini, T., Santiago Garcia, J., Richer, J. S., Bachiller, R., \& Tafalla, M. 2007, A\&A, 462, 163

Olofsson, H., Hjalmarson, A., \& Rydbeck, O. E. H. 1981a, A\&A, 100, L30

Olofsson, H., Rydbeck, O. E. H., Lane, A. P., \& Predmore, C. R. 1981b, ApJ, 247, L81

Penzias, A. A. 1981, ApJ, 249, 513

Reid, M. J., Menten, K. M., Greenhill, L. J., \& Chandler, C. J. 2007, ApJ, 664, 950

Schilke, P., Walmsley, C. M., Pineau des Forets, G., \& Flower, D. R. 1997a, A\&A, 321, 293

Schilke, P., Groesbeck, T. D., Blake, G. A., \& Phillips, T. G. 1997b, ApJS, 108, 301

Schilke, P., Benford, D. J., Hunter, T. R., Lis, D. C., \& Phillips, T. G. 2001, ApJS, 132, 281

Snyder, L. E., \& Buhl, D. 1974, ApJ, 189, L31

Thaddeus, P., Mather, J., Davis, J. H., \& Blair, G. N. 1974, ApJ, 192, L33

Tipping, R. H., \& Chackerian, C., Jr. 1981, Journal of Molecular Spectroscopy, 88, 352

Wolff, R. S. 1980, ApJ, 242, 1005

Wright, M. C. H., Plambeck, R. L., Mundy, L. G., \& Looney, L. W. 1995, ApJ, 455, L185

Zapata, L. A., Leurini, S., Menten, K. M., Schilke, P., Rolffs, R., \& Hieret, C. 2008, AJ, 136, 1455

Zombeck, M. 2007, Handbook of Space Astronomy and Astrophysics: Third Edition, by Martin Zombeck. ISBN-10 0521-78242-2 (HB); ISBN-13 781-0-521-78242-5 (HB). Published by Cambridge University Press, Cambridge, UK, 2007. 